\title{
Calculation of pseudoscalar disconnected quark loops in Lattice QCD
}

\section{Zhen Cheng*}

Department of Physics, Zhejiang University, Hangzhou, 310027, P.R. China

E-mail: zjuercz@zju.edu.cn

\section{Guang-yi Xiong}

Department of Physics, Zhejiang University, Hangzhou, 310027, P.R. China

E-mail: xionggyezju.edu.cn

\section{Jian-bo Zhang}

Department of Physics, Zhejiang University, Hangzhou, 310027, P.R. China

E-mail: jbzhang08@zju.edu.cn

\begin{abstract}
The evaluation of disconnected quark loops are often required in the lattice QCD calculations and very computer time consuming. To compute these diagrams, stochastic noise methods are generally used. However, stochastic estimation has large error in the calculations of the pseudoscalar disconnected diagrams. We use a symmetric multi-probing method (SMP) to estimate these disconnected quark loops. We compare the SMP method to the $Z(2)$ noise method for computing the pseudoscalar disconnected quark loops on quenched lattice $\mathrm{QCD}$ ensemble with lattice volume $12^{3} \times 24$ at lattice spacing $a \approx 0.1 \mathrm{fm}$. It is found that the SMP method is very suitable for the calculation of the pseudoscalar disconnected quark loops.
\end{abstract}

37th International Symposium on Lattice Field Theory - Lattice2019

16-22 June 2019

Wuhan, China

${ }^{*}$ Speaker. 


\section{Introduction}

Lattice QCD, first introduced by K. Wilson in 1974 [1], is a gauge invariant and nonperturbative regularization scheme for QCD. Many quantities can be obtained from first principles by using a finite space-time lattice to simulate the interactions between quarks and gluons. One of the most challenging problems in Lattice QCD is the calculation of diagrams with disconnected quark loops. Many physical quantities in QCD are affected by such loops, such as pi-NN coupling and pion polarizability, nucleon electromagnetic form factors, the mass of pseudoscalar flavor-singlet mesons, the strangeness and charm contents of the nucleon, hadronic scattering lengths and structure functions, and electron or muon hadronic $g-2$ loop contributions. In lattice QCD, the expectation value of disconnected quark loops can be written as [2]

$$
\langle\bar{\psi} \Gamma \psi\rangle=-\operatorname{Tr}\left(\Gamma M^{-1}\right),
$$

where $\Gamma \in\left\{\mathbb{1}, \gamma_{\mu}, \gamma_{5}, \gamma_{5} \gamma_{\mu}, \sigma_{\mu v}, \mu, v=1,2,3,4\right\}$, and $M$ is the Dirac fermion matrix operator. We will use Wilson's Dirac operator in this work. This Dirac operator can be written as

$$
\begin{gathered}
M=\mathbb{1}-\kappa D, \quad \kappa=\frac{1}{2(a m+4)} \\
D(n \mid m)_{\substack{\alpha \beta \\
a b}}=\sum_{\mu=1}^{4}\left(\mathbb{1}-\gamma_{\mu}\right)_{\alpha \beta} U_{\mu}(n)_{a b} \delta_{n+\hat{\mu}, m}+\sum_{\mu=1}^{4}\left(\mathbb{1}+\gamma_{\mu}\right)_{\alpha \beta} U_{-\mu}(n)_{a b} \delta_{n-\hat{\mu}, m},
\end{gathered}
$$

where $a$ is the lattice spacing. $D$ is called hopping matrix and the real number $\kappa$ is the hopping parameter [3]. In the calculation of the disconnected diagrams, we need to solve the equation

$$
M x=b,
$$

where $M$ is the Dirac matrix with dimension $K \times K, b$ is the source vector of dimension $K \times 1$. The solution of this equation is

$$
x=M^{-1} b
$$

The evaluation of disconnected quark loops requires $M^{-1}$ connecting arbitrary pairs of lattice points. Wilson's Dirac matrix is a large sparse matrix and its typical dimension $K$ is $10^{6}$ up to $10^{9}$, direct evaluation of $M^{-1}$ using point source (p-s) would be prohibitively expensive, both in terms of computer time and of memory.

In order to calculate disconnected quark loops, specific techniques must be introduced. Unbiased noise methods are traditionally used to estimate the inverse matrix [4]. The truncated solver method (TSM) [5], and spin explicit method (SEM) [6] are also introduced to reduce the stochastic error. A probing technique is also a way to deal with this problem [7]. However, we found that all stochastic methods have too large errors in the calculation of the pseudoscalar disconnected quark loops $\operatorname{Tr}\left(\gamma_{5} M^{-1}\right)$. We introduce a new method, called the symmetric multi-probing source (SMP) method, to calculate the pseudoscalar disconnected quark loops. We compared SMP method to $Z(2)$ noise method, based on the point source results. 


\section{Methods}

\section{1 $Z(2)$ noise method}

First, we briefly review $Z(2)$ noise method. Assuming that we have $L$ column noise vectors $b^{1}, b^{2}, b^{3}, \ldots, b^{L}$, these vectors have two properties as follows [4],

$$
<b_{i}>=\frac{1}{L} \sum_{l=1}^{L} b_{i}^{l}=0, \quad<b_{i} b_{j}>=\frac{1}{L} \sum_{l=1}^{L} b_{i}^{l} b_{j}^{l}=\delta_{i j}
$$

where $b_{i}^{l}$ is the i-th entry in the noise entry $l$. The stochastic average $\langle\cdots\rangle$ goes over the ensemble of the noise vectors $L$.

$Z(2)$ noise vectors are used in eq.(1.4), we can obtain

$$
x_{i}^{l}=\sum_{k} M_{i k}^{-1} b_{k}^{l} .
$$

Any inverse matrix element, $M_{i j}^{-1}$, is given as follow

$$
<b_{j} x_{i}>=\sum_{k} M_{i k}^{-1}<b_{j} b_{k}>=M_{i j}^{-1} .
$$

The variance of the $Z(2)$ noise method is [8]

$$
\sigma^{2} \equiv \frac{1}{L} \sum_{i \neq j}^{K}\left|M_{i j}^{-1}\right|^{2}
$$

It can be seen that the stochastic error of the $Z(2)$ noise estimate results only from the offdiagonal entries of the inverse matrix.

Therefore, we can get $\operatorname{Tr}\left(\Gamma M^{-1}\right)$

$$
\operatorname{Tr}\left(\Gamma M^{-1}\right)=\sum_{j} \Gamma M_{j j}^{-1}=\sum_{i} \frac{1}{L} \sum_{l} b_{i}^{l}\left(\Gamma x_{i}^{l}\right) .
$$

\subsection{SMP method}

The symmetric multi-probing source vector $\phi_{P}$ is introduced as follows [9]

$$
\phi_{P}(S(x, P), \alpha, a)=\sum_{y \in S(x, P)} \psi(y, \alpha, a),
$$

where $\alpha$ is the Dirac index, $a$ the color index. $S(x, P)$ represents the sites with the same color of $x$ obtained by the symmetric coloring scheme $P\left(\frac{n_{s}}{d}, \frac{n_{s}}{d}, \frac{n_{s}}{d}, \frac{n_{t}}{d}\right.$, mode $)$, where $n_{s}$ and $n_{t}$ are spatial and temporal sizes of lattice respectively, $d$ is the distance parameter, mode $=0,1,2$ corresponds to Normal, Split and Combined mode. $x$ is the seed site at $\left(x_{1}, x_{2}, x_{3}, x_{4}\right)$ and $y$ a lattice site. $\psi$ is a normalized p-s vector. The number of SMP sources $N_{\mathrm{SMP}}$ on lattice is

$$
N_{\mathrm{SMP}}= \begin{cases}12 d^{4} & \text { mode }=0 \\ 24 d^{4} & \text { mode }=1 \\ 6 d^{4} & \text { mode }=2\end{cases}
$$


Appling SMP source in eq.(1.4) to calculate the trace of $\Gamma M^{-1}$, we can obtain

$$
\begin{aligned}
\operatorname{Tr}\left(\Gamma M^{-1}\right)_{\mathrm{SMP}}= & \sum_{x, \alpha, a} \psi(x, \alpha, a)\left(\Gamma M^{-1}\right) \phi_{P}(S(x, P), \alpha, a) \\
= & \sum_{x, \alpha, a} \psi(x, \alpha, a)\left(\Gamma M^{-1}\right) \psi(x, \alpha, a) \\
& +\sum_{y \in S(x, P)}^{y \neq x} \sum_{\alpha, a} \psi(x, \alpha, a)\left(\Gamma M^{-1}\right) \psi(y, \alpha, a) \\
= & \operatorname{Tr}\left(\Gamma M^{-1}\right)+\sum_{y \in S(x, P)}^{y \neq x} \sum_{\alpha, a} \psi(x, \alpha, a)\left(\Gamma M^{-1}\right) \psi(y, \alpha, a),
\end{aligned}
$$

where the second term $\sum_{y \in S(x, P)}^{y \neq x} \sum_{\alpha, a} \psi(x, \alpha, a)\left(\Gamma M^{-1}\right) \psi(y, \alpha, a)$ is the sum of some off-diagonal elements of $\Gamma M^{-1}$. This term can be regarded as the error in the calculation of $\operatorname{Tr}\left(\Gamma M^{-1}\right)$. When we choose the proper scheme $P$ of SMP source, the error will be quite small. In this case, we can neglect the error term and get

$$
\operatorname{Tr}\left(\Gamma M^{-1}\right) \approx \operatorname{Tr}\left(\Gamma M^{-1}\right)_{\mathrm{SMP}}
$$

\section{Simulation details}

It is extremely time-consuming to solve eq. (1.4) if $b$ is the point source vector and running over all the lattice sites on large lattice. In this work, we will use point source method to evaluate $\operatorname{Tr}\left(\Gamma M^{-1}\right)$ and treat them as the exact values for comparison. Considering huge computer time, we only analyse ensembles of Iwasaki pure gauge configurations on $L_{\sigma}^{3} \times L_{\tau}=12^{3} \times 24$. The lattice spacing of the ensembles is $a \approx 0.1 \mathrm{fm}$. The analysis is performed on 10 configurations at $\kappa=0.151$, corresponding to $m_{\pi} \approx 488 \mathrm{MeV}$. We only show the results of $\operatorname{Tr}\left(\gamma_{5} M^{-1}\right)$ in this work.

We know that identity $M=\gamma_{5} M^{\dagger} \gamma_{5}[10]$, so that

$$
\operatorname{Tr}\left(\gamma_{5} M^{-1}\right)=\operatorname{Tr}\left(\gamma_{5} \gamma_{5}\left(M^{-1}\right)^{\dagger} \gamma_{5}\right)=\operatorname{Tr}\left(\left(M^{-1}\right)^{\dagger} \gamma_{5}\right)=\operatorname{Tr}\left(\left(\gamma_{5} M^{-1}\right)^{\dagger}\right) .
$$

Therefore, $\operatorname{Tr}\left(\gamma_{5} M^{-1}\right)$ is real number. We only focus on absolute error and it is defined as

$$
\Delta r=\left|r_{p}-r_{m}\right|,
$$

where $r_{p}$ is the result of point source, and $r_{m}$ is the value of other method different from the point source. All results in this research are compared with the result of the point source for each configuration (conf.).

\section{Results and analyses}

The results of $\operatorname{Tr}\left(\gamma_{5} M^{-1}\right)$ for SMP and $Z(2)$ noise methods are shown In Tables 1 and 2, respectively. The absolute errors of $\operatorname{Tr}\left(\gamma_{5} M^{-1}\right)$ are also presented in the following Figures 1 and 2. These show that SMP method has great advantage in the calculation of $\operatorname{Tr}\left(\gamma_{5} M^{-1}\right)$. 


\begin{tabular}{|c|c|c|c|c|c|c|c|}
\hline conf. & $\mathrm{p}-\mathrm{s}$ & $N=15552$ & $N=3072$ & $N=1536$ & $N=384$ & $N=192$ & $N=96$ \\
\hline 1 & -18.94 & -23.51 & -41.58 & -41.64 & -27.07 & -58.30 & -169.71 \\
\hline 2 & 202.86 & 196.47 & 198.40 & 212.43 & 318.32 & 321.95 & 242.65 \\
\hline 3 & 183.32 & 181.34 & 173.99 & 174.20 & 101.93 & 122.88 & -3.02 \\
\hline 4 & -170.65 & -177.74 & -194.22 & -201.41 & -132.14 & -140.37 & -167.82 \\
\hline 5 & -586.77 & -596.83 & -626.25 & -627.35 & -534.12 & -532.24 & -509.32 \\
\hline 6 & 74.85 & 60.69 & 53.81 & 65.54 & 56.35 & 50.80 & -106.45 \\
\hline 7 & -288.10 & -298.31 & -307.69 & -304.96 & -231.95 & -302.11 & -458.16 \\
\hline 8 & 141.12 & 126.73 & 105.84 & 93.16 & 223.25 & 167.02 & 91.50 \\
\hline 9 & -338.56 & -329.98 & -334.35 & -327.84 & -263.26 & -317.34 & -477.56 \\
\hline 10 & -223.22 & -233.52 & -207.10 & -200.56 & -187.40 & -192.48 & -250.08 \\
\hline
\end{tabular}

Table 1: Results of $\operatorname{Tr}\left(\gamma_{5} M^{-1}\right)$ for $Z(2)$ noise method. $N$ represents the number of source vectors. It shows that $Z(2)$ noise method has too large error in the calculation of $\operatorname{Tr}\left(\gamma_{5} M^{-1}\right)$, especially for the small $N$.

\begin{tabular}{|c|c|c|c|c|c|c|c|}
\hline conf. & $\mathrm{p}-\mathrm{s}$ & $\begin{array}{c}N=15552 \\
(6,0)\end{array}$ & $\begin{array}{c}N=3072 \\
(4,0)\end{array}$ & $\begin{array}{c}N=1536 \\
(4,2)\end{array}$ & $\begin{array}{c}N=384 \\
(2,1)\end{array}$ & $\begin{array}{c}N=192 \\
(2,0)\end{array}$ & $\begin{array}{c}N=96 \\
(2,2)\end{array}$ \\
\hline 1 & -18.94 & -18.37 & -22.18 & -15.61 & -10.08 & -21.66 & -22.73 \\
\hline 2 & 202.86 & 203.21 & 202.90 & 212.26 & 192.83 & 184.64 & 188.23 \\
\hline 3 & 183.32 & 184.33 & 181.43 & 180.09 & 178.41 & 178.20 & 148.52 \\
\hline 4 & -170.65 & -170.89 & -163.42 & -160.16 & -186.75 & -172.39 & -206.26 \\
\hline 5 & -586.77 & -583.80 & -563.81 & -555.51 & -559.26 & -569.70 & -519.76 \\
\hline 6 & 74.85 & 73.18 & 73.35 & 75.99 & 81.00 & 96.71 & 124.26 \\
\hline 7 & -288.10 & -290.81 & -288.23 & -284.46 & -291.66 & -280.63 & -271.28 \\
\hline 8 & 141.12 & 141.86 & 137.16 & 138.44 & 153.09 & 167.57 & 164.22 \\
\hline 9 & -338.58 & -335.66 & -342.16 & -329.44 & -338.10 & -332.02 & -351.82 \\
\hline 10 & -223.22 & -220.96 & -224.15 & -221.93 & -237.32 & -218.63 & -222.10 \\
\hline
\end{tabular}

Table 2: Results of $\operatorname{Tr}\left(\gamma_{5} M^{-1}\right)$ for SMP method. Components in brackets in first row represent the parameters $d$ and mode of the SMP sources, for example, $(6,0)$ denotes $d=6$ and mode $=0$. As the number of sources increases, the results of SMP method become more and more accurate.

In Fig. 1, the absolute errors of SMP and Z(2) noise methods are shown. It indicates that the absolute errors of SMP and $Z(2)$ noise methods decrease with the increase of the number of the sources. However, SMP method has much more smaller absolute errors than $Z(2)$ noise method. 

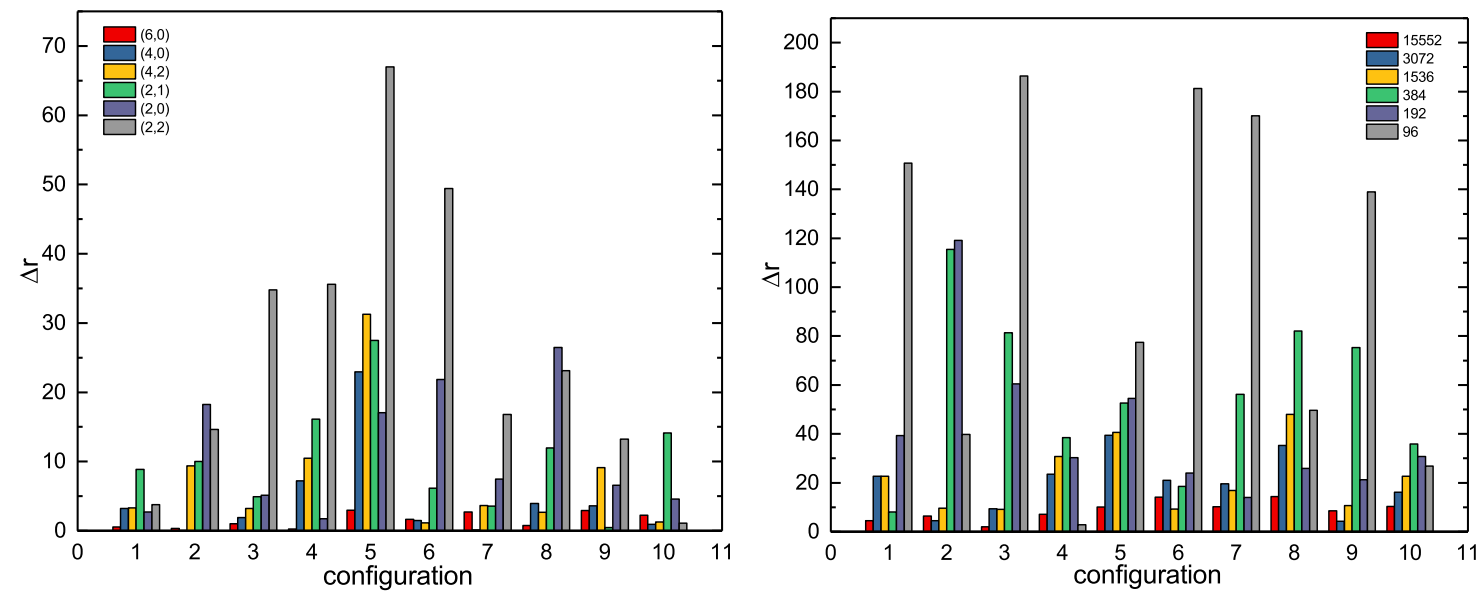

Figure 1: Absolute errors for different method. Left: Absolute errors of SMP method. Right: Absolute errors of $Z(2)$ noise method.
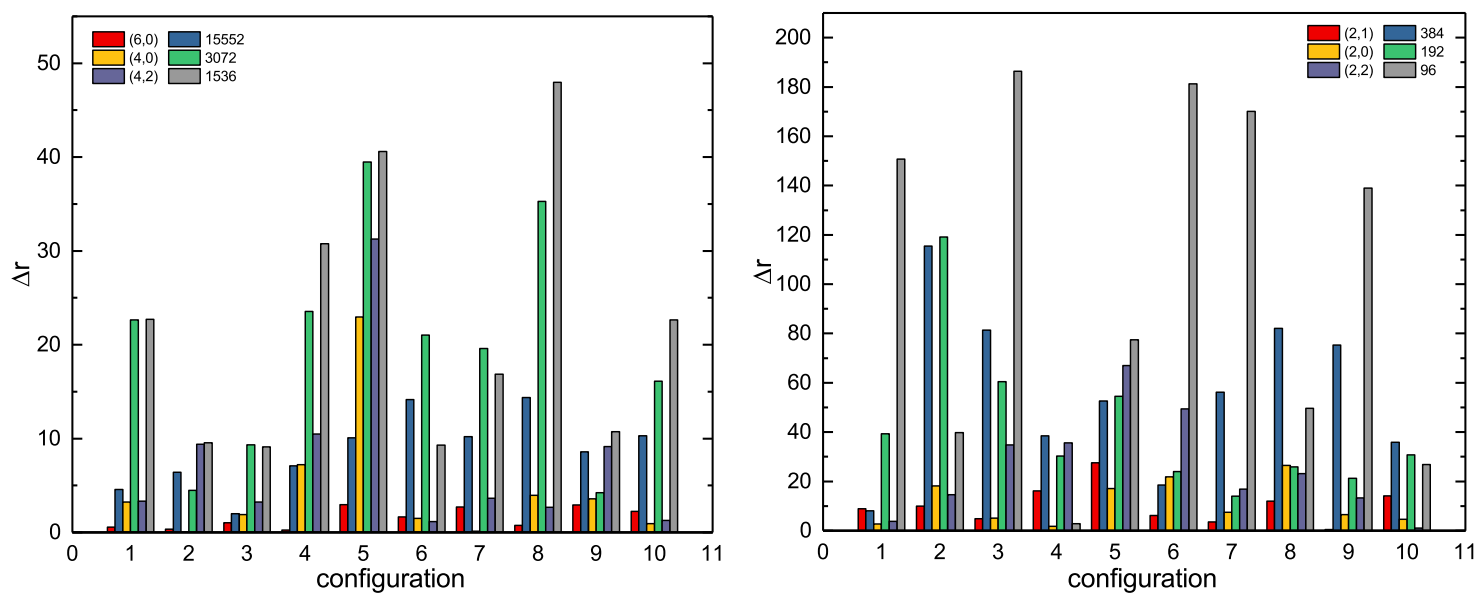

Figure 2: Absolute errors of SMP and Z(2) noise methods. SMP method has much smaller absolute errors than $Z(2)$ noise method. Brackets after the legends show the parameters of the SMP source vectors, and numbers after the legends indicate the number of $Z(2)$ noise source vectors.

In Fig. 2, we compare the results of the SMP method with that of Z(2) noise method with same number of source vectors. Absolute errors of SMP method are about an order of magnitude smaller than that of $Z(2)$ noise method.

All results indicate that the $Z(2)$ noise method has larger error than SMP method when they has the same number of source vectors in the calculation of $\operatorname{Tr}\left(\gamma_{5} M^{-1}\right)$. SMP method has great advantage on the estimation of $\operatorname{Tr}\left(\gamma_{5} M^{-1}\right)$. It also shows that the results of SMP method are quite precise when the parameter $d$ is large enough.

\section{Conclusions}

We have studied the calculation of the pseudoscalar disconnected quark loops by the SMP and $\mathrm{Z}(2)$ noise methods. Absolute errors of the SMP and $Z(2)$ noise methods decrease with the 
increase of the number of sources in the evaluation of the pseudoscalar disconnected loops. We found that the SMP method can improve the calculation precision by one order of magnitude in the estimation of $\operatorname{Tr}\left(\gamma_{5} M^{-1}\right)$. All results show that the SMP method is the better way to calculate $\operatorname{Tr}\left(\gamma_{5} M^{-1}\right)$.

\section{Acknowledgments}

This work was run on Tianhe-2 supercomputer at NSCC in Guangzhou.

\section{References}

[1] Kenneth G. Wilson. Confinement of quarks. Phys. Rev. D, 10:2445-2459, Oct 1974.

[2] Suman Baral, Travis Whyte, Walter Wilcox, and Ronald B. Morgan. Disconnected Loop Subtraction Methods in Lattice QCD. Comput. Phys. Commun., 241:64-79, 2019.

[3] Christof Gattringer and Christian B. Lang. Quantum chromodynamics on the lattice. Lect. Notes Phys., 788:1-343, 2010.

[4] Shao-Jing Dong and Keh-Fei Liu. Stochastic estimation with Z(2) noise. Phys. Lett., B328:130-136, 1994.

[5] Gunnar S. Bali, Sara Collins, and Andreas Schafer. Effective noise reduction techniques for disconnected loops in Lattice QCD. Comput. Phys. Commun., 181:1570-1583, 2010.

[6] J. Viehoff. News on disconnected diagrams. Nucl. Phys. Proc. Suppl., 73:856-858, 1999.

[7] Tang Jok Man and Saad Yousef. A new method for computing the diagonal of a matrix inverse. HKIE Transactions, 17(4):69-72, 2010.

[8] C. Thron, S. J. Dong, K. F. Liu, and H. P. Ying. Padé-Z $Z_{2}$ estimator of determinants. Phys. Rev. D, 57:1642-1653, Feb 1998.

[9] Guang-Yi Xiong, Jian-Bo Zhang, and You-Hao Zou. Evaluating the topological charge density with the symmetric multi-probing method. Chin. Phys., C43(3):033102, 2019.

[10] Walter Wilcox. Noise methods for flavor singlet quantities. In Numerical challenges in lattice quantum chromodynamics. Proceedings, Joint Interdisciplinary Workshop, Wuppertal, Germany, August 22-24, 1999, pages 127-141, 1999. 